\title{
Atomic structure and segregation in alkali-metal heteroclusters
}

\author{
A. Mañanes \\ Departamento de Física Moderna, Universidad de Cantabria, Santander, Spain \\ M. P. Iñiguez, M. J. López, and J. A. Alonso \\ Departamento de Física Teórica y Física Atómica, Molecular y Nuclear Universidad de Valladolid, Valladolid, Spain
}

(Received 26 February 1990)

\begin{abstract}
The ground-state atomic and electronic distributions in $\mathrm{Na}_{m} \mathrm{Cs}_{n}$ clusters with composition $m=n$ and $m=2 n$ have been calculated by minimizing the total cluster energy using the density-functional formalism. The approximation is made by replacing the total external potential of the ions by its spherical average around the cluster center during the iterative process of solving the Kohn-Sham equations for each geometry tested. In the size range studied here (up to 90 atoms per cluster), the cluster is composed of well-separated homoatomic $\mathrm{Na}$ and $\mathrm{Cs}$ shells, the external one always being a Cs shell. We have also found that the cohesive energy goes rapidly to the bulk limit. An analysis of the geometries shows strong cluster reconstruction with increasing size. By comparing the geometry of pure $\mathrm{Na}_{n}$ with that of the $\mathrm{Na}_{n}$ core in $\mathrm{Na}_{n} \mathrm{Cs}_{n}$ for clusters formed by only an inner $\mathrm{Na}$ layer and an outer $\mathrm{Cs}$ layer, we have observed that the $\mathrm{Na}_{n}$ core adopts a geometry different in most cases from that of the free $\mathrm{Na}_{n}$ cluster, and such that the number of faces of the polyhedron formed by the $\mathrm{Na}_{n}$ core is as close as possible to the number of external Cs atoms, in order to accomodate these Cs atoms on top of the faces of the polyhedron.
\end{abstract}

\section{INTRODUCTION}

Clusters with an atomic content below, say, 100 atoms, have a substantial part of them on the surface. Their atomic arrangements are usually different from their bulk counterparts due to the surface effect. When the cluster size decreases, the geometry depends more sensitively on the detailed electronic structure and bonding. In pure bulk metals we know that only a few types of crystalline structures occur; in contrast, clusters with few atoms reconstruct their geometries with increasing size in a way not yet known.

The delocalized character of the electrons in alkalimetal clusters and heteroclusters has been inferred from experiments. ${ }^{1}$ A model of electrons in a spherical or an ellipsoidal potential well due to a positive jellium background accounts for many experimental results. ${ }^{1-4}$ Properties depending on the discrete nature of the ions are evidently outside the scope of the jellium model.

In a study of the liquid-vapor interface of the $\mathrm{Na}$ metal and Na-Cs alloys, Rice and co-workers ${ }^{5-8}$ have performed Monte Carlo simulations for very large aggregates in the size range between 100 and 1000 atoms. These authors have shown that a pronounced stratification of the atomic distribution develops near the surface, with a layer spacing of about one atomic diameter. Furthermore, segregation of $\mathrm{Cs}$ to the surface was found in the simulations for the Na-Cs alloy.

In small alkali-metal heteroclusters, with most atoms at or very near to the surface, it is expected that stratification and segregation effects will dominate the entire cluster structure. Some preliminary results presented by the authors ${ }^{9}$ have shown that the cluster $\mathrm{Na}_{10} \mathrm{Cs}_{10}$ is formed by an inner $\mathrm{Na}$ shell surrounded by a $\mathrm{Cs}$ shell.
Using the Car-Parrinello method, Ballone et al. ${ }^{10}$ have found segregation of potassium to the surface of a $\mathrm{Na}_{10} \mathrm{~K}_{10}$ cluster.

Relevant questions in this area are the following: How does the segregation effect depend on cluster size? Evidently, segregation is a surface effect. But what happens when one looks at the whole cluster? Is there random mixing of $\mathrm{Na}$ and Cs? Or, rather, do ordering or full phase separation occur? Bulk Na-Cs alloys are miscible in the liquid state. ${ }^{11}$ In the solid state a single intermetallic compound, of composition $\mathrm{Na}_{2} \mathrm{Cs}$, forms at low temperature, but solid solutions do not exist. This indicates the importance of the temperature for mixing. In summary, it should be interesting to know if the miscibility properties change in microalloys with fewer than 100 atoms. In this work we study the atomic arrangements of $\mathrm{Na}_{m} \mathrm{Cs}_{n}$ heteroclusters with $m=n$ and $2 n$, and with a total number of atoms up to $N=n+m=90$, paying particular attention to questions like mixing, ordering, segregation, and relative stability of the clusters as a function of size and magic numbers.

\section{MODEL}

In this section we give a brief sketch of the computational method used to obtain the ground-state geometry, and the corresponding energy, of the clusters.

First of all, the atoms are placed at random positions, and the valence-electron distribution is self-consistently calculated by minimization of the total energy, according to the Kohn-Sham version of density-functional theory ${ }^{12}$ using the local-density approximation for exchange and correlation effects. ${ }^{13}$ At this moment, the net forces at the ionic sites are nonzero, and then the ions are allowed 
to move a small distance in the force directions. The electron distribution is computed for this new atomic arrangement, and the whole cycle is iterated until a minimum of the total energy with respect to all ionic coordinates is obtained, that is, until all the forces vanish. As the only guarantee for reaching the absolute minimum, and not a relative one, the whole process is repeated for a sufficient number of random initial configurations of the atoms (in practice, this number has been taken to be larger than 40).

Empty-core model potentials ${ }^{14}$ are used to describe the pseudopotentials of the individual ions. The empty-core radii, $r_{c}(\mathrm{Na})=1.74$ a.u. and $r_{c}(\mathrm{Cs})=2.74$ a.u., lead to the experimental ionization potentials in the free-atom limit for the same density-functional method.

An essential approximation of the method is that the total ionic potential due to the ions at positions $\mathbf{R}_{1}$,

$$
V_{I}(\mathbf{r})=\sum_{i} v\left(\left|\mathbf{r}-\mathbf{R}_{i}\right|\right),
$$

is substituted by its spherical average around the cluster center (center of ionic charge), $V_{I}^{\text {av }}(r)$, in the process of integrating the Kohn-Sham equations. For this reason we call this method the spherically averaged pseudopotential (SAPS) method. ${ }^{15}$ However, the exact ion-ion Coulombic repulsion is evaluated in our calculations, which makes the total energy a function dependent on the precise location of the atoms.

Additional details concerning the density-functional formalism used here can be consulted in Ref. 15, where applications to different pure-metal clusters have been done.

\section{RESULTS}

\section{A. Layering, segregation, and growth}

Let us consider first the main features of the atomic distribution in $\mathrm{Na}_{m} \mathrm{Cs}_{n}$ clusters with $m=n$ and $2 n$ and a total number of atoms $N \leq 90$. A common characteristic for the two concentrations and all sizes is the formation of homoatomic shells, that is, separated shells of $\mathrm{Na}$ and Cs atoms, respectively, with the most external shell always occupied by $\mathrm{Cs}$ atoms. The most salient features concerning the formation and evolution of these shells with cluster size can be observed in Fig. 1. The radial distribution of atoms (with respect to the center of ionic charge of the cluster) has been plotted for a few representative cases $\left(N=8,20,34,40,58\right.$, and 90 for $\mathrm{Na}_{n} \mathrm{Cs}_{n}$ and $N=9,21,39$, and 90 for $\mathrm{Na}_{2 n} \mathrm{Cs}_{n}$ ). Clusters up to about $N=42$ have two homoatomic layers (for both concentrations), the inner one formed by the $\mathrm{Na}$ atoms and the outer one by the Cs atoms. The definition of the Na layer is more ambiguous than that of the Cs layer, this one being appreciably thinner. For $\mathrm{Na}_{n} \mathrm{Cs}_{n}$ it is perhaps more realistic to describe the distribution of the $\mathrm{Na}$ atoms by a well-defined shell only in the restricted size range $N \leq 26$. Instead, from $N=28$ up to $N=44$ there are a few intermediate $\mathrm{Na}$ atoms halfway between the internal $(\mathrm{Na})$ and external (Cs) shells (see, for instance, $\mathrm{Na}_{20} \mathrm{Cs}_{20}$ in Fig. 1).
For $\mathrm{Na}_{2 n} \mathrm{Cs}_{n}$ one observes an appreciable broadening of the whole $\mathrm{Na}$ shell with respect to $\mathrm{Na}_{n} \mathrm{Cs}_{n}$. Larger clusters with $46 \leq N \leq 60$ develop a Cs layer in the inner region. Then their configuration is that of a $\mathrm{Na}$ region with a large dispersion of radial distances bound on the inside by one Cs shell and on the outside by another, more populated, Cs shell. It is noticeable that the cluster size $N$ at which the inner Cs shell appears is about the same for the two concentrations studied here. One could have expected some difference due to concentration because the Cs$\mathrm{Na}-\mathrm{Cs}$ stratification can be considered as a precursor of the tendency to superlattice formation in the bulk solid alloy and the only stable intermetallic compound that has the $\mathrm{Na}_{2} \mathrm{Cs}$ composition. ${ }^{11}$ Many more atoms are needed, however, to resemble closely the bulk behavior. Finally, the figure also shows the atomic distributions for the two concentrations at $N=90$. In this case a new $\mathrm{Na}$ shell appears in the inner region of $\mathrm{Na}_{60} \mathrm{Cs}_{30}$ but not in $\mathrm{Na}_{45} \mathrm{Cs}_{45}$. This difference is due to the different concentration, and it suggests that the stoichiometry $\mathrm{Na}_{2} \mathrm{Cs}$ develops ordering more easily than the $\mathrm{NaCs}$ stoichiometry, which is in agreement with the situation observed in the bulk phase. ${ }^{11}$ In summary, we can look at the process of cluster growth as the successive formation of inner homoatomic shells, at least in the size range $N<100$.

Besides the existence of homoatomic shells, we observe, for most clusters with $N>10$, a strong tendency to find one single atom at the cluster center. For $\mathrm{Na}_{n} \mathrm{Cs}_{n}$ the central atom is $\mathrm{Na}$ up to $N=26$, whereas at larger sizes the nature of the central atom is anticorrelated with that of the innermost shell; that is, the central atom is Cs when the innermost shell is formed by $\mathrm{Na}$ atoms and vice versa. For $\mathrm{Na}_{2 n} \mathrm{Cs}_{n}$ the central atom is $\mathrm{Na}$ with very few exceptions.

The valence-electron density is also plotted in Fig. 1, in units of the average bulk electron density $\rho_{\mathrm{av}}$, defined as $\rho_{\mathrm{av}}=1 / \Omega_{\mathrm{av}}$, where $\Omega_{\mathrm{av}}=[m \Omega(\mathrm{Na})+n \Omega(\mathrm{Cs})] / N, \Omega(\mathrm{Na})$ and $\Omega(\mathrm{Cs})$ being the volumes per atom in bulk $\mathrm{Na}$ and $\mathrm{Cs}$, respectively.

The (average) radii of the shells and their widths (measured by the standard deviations) are given in Fig. 2 for $\mathrm{Na}_{n} \mathrm{Cs}_{n}$. The width of the surface (Cs) shell never exceeds 1 a.u. In contrast, the dispersion in the $\mathrm{Na}$ shell is larger, about 2 a.u. in some cases, and it would increase further if the intermediate $\mathrm{Na}$ atoms in the size range $N=28-44$ (these have been omitted from the figure) were ascribed to this shell. The radius of the surface shell, which is a measure of the cluster size, is a linear function of $N^{1 / 3}$ with a slope approximately equal to $r_{s}^{*}$ $\left[r_{s}^{*}=\left(3 \Omega_{\mathrm{av}} / 4 \pi\right)^{1 / 3}\right]$. Radii for $\mathrm{Na}_{2 n} \mathrm{Cs}_{n}$ are not shown in the figure, but it is interesting to note that the radius of the external Cs shell is very similar for the two concentrations at each size, considering the large atomic size difference, between $\mathrm{Na}$ and $\mathrm{Cs}$.

The distribution of Cs atoms in $\mathrm{Na}_{n} \mathrm{Cs}_{n}$ is given in Fig. 3. The majority of Cs atoms is in the surface shell. First, the number of atoms in the surface shell increases steeply with $n$, but later this number grows at a lower rate due to the formation of the inner Cs shell.

Previous calculations ${ }^{15,16}$ for homoatomic $\mathrm{Na}_{n}$ and $\mathrm{Cs}_{n}$ 
(a)
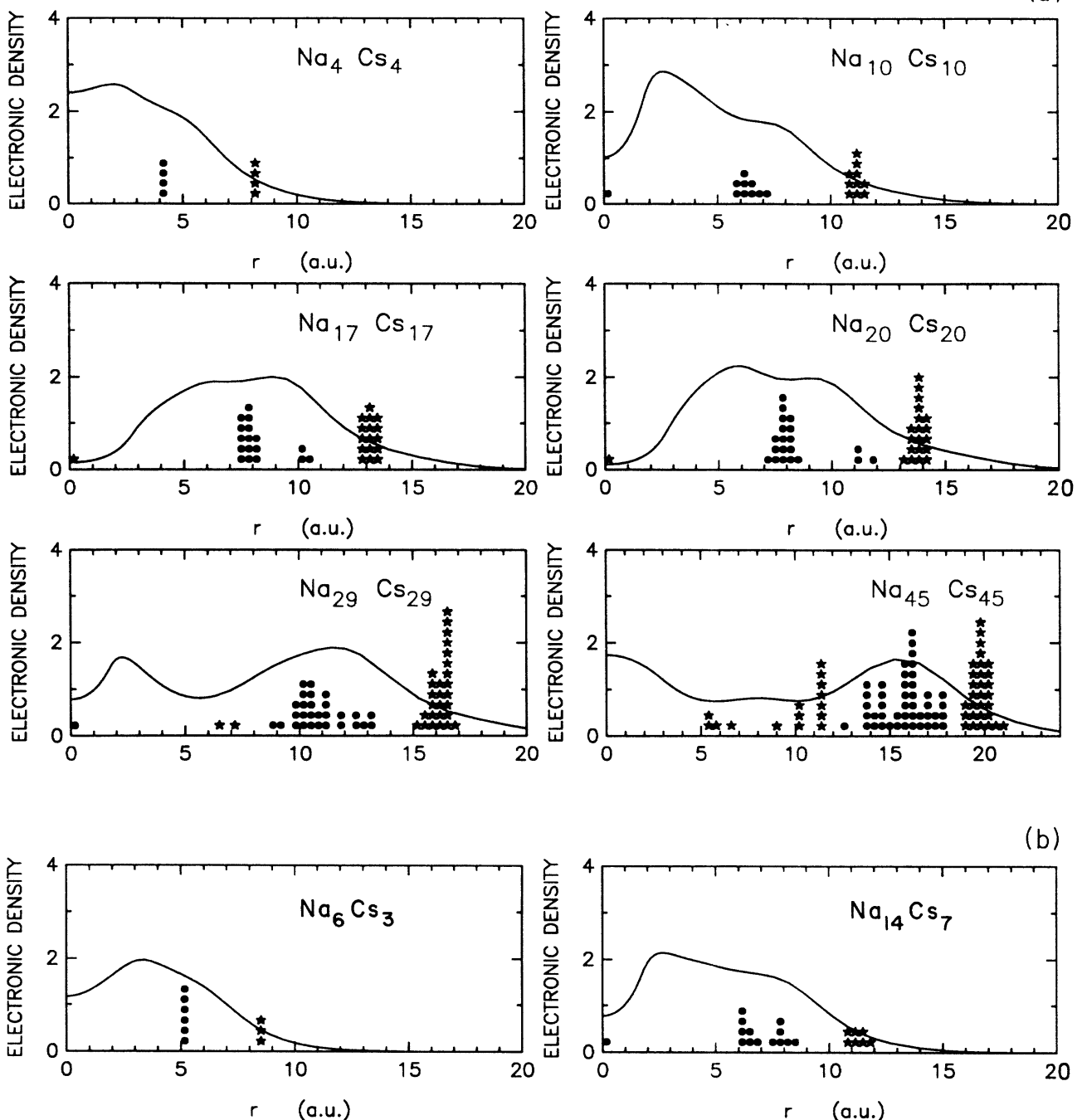

(b)
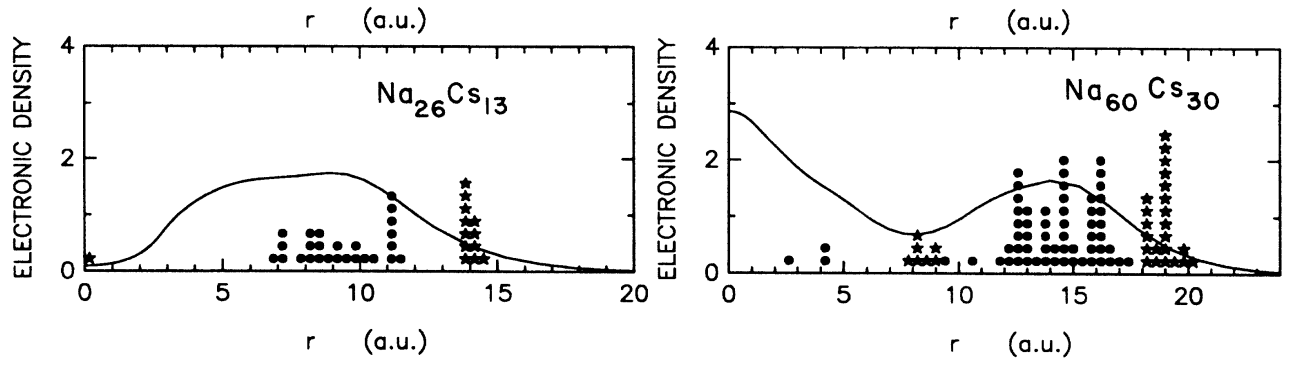

FIG. 1. Radial distribution of atoms (with respect to the center of ionic charge of the cluster). Stars indicate Cs atoms and circles $\mathrm{Na}$ atoms. The electron density is also plotted in units of the average bulk electron density $\rho_{\mathrm{av}}$, defined $\rho_{\mathrm{av}}=1 / \Omega_{\mathrm{av}}$ with $\Omega_{\mathrm{av}}=[m \Omega(\mathrm{Na})+n \Omega(\mathrm{Cs})] / N$, where $\Omega(\mathrm{Na})$ and $\Omega(\mathrm{Cs})$ are volumes per atom in the pure bulk metals. (a) $\mathrm{Na}_{4} \mathrm{Cs}_{4}, \mathrm{Na}_{10} \mathrm{Cs}_{10}, \mathrm{Na}_{17} \mathrm{Cs}_{17}$, $\mathrm{Na}_{20} \mathrm{Cs}_{20}, \mathrm{Na}_{29} \mathrm{Cs}_{29}$, and $\mathrm{Na}_{45}, \mathrm{Cs}_{45}$. (b) $\mathrm{Na}_{6} \mathrm{Cs}_{3}, \mathrm{Na}_{14} \mathrm{Cs}_{7}, \mathrm{Na}_{26} \mathrm{Cs}_{13}$, and $\mathrm{Na}_{60} \mathrm{Cs}_{30}$.

clusters using the SAPS method have also produced a structure formed by atomic layers. In the case of sodium clusters, there is one single layer for $n<7$, one layer plus a central atom for $7 \leq n \leq 19$ (except for $\mathrm{Na}_{8}$ ), ${ }^{17}$ and two layers for $20 \leq n \leq 50$. The situation for $\mathrm{Cs}_{n}$ is very similar ${ }^{16}$ to the small difference that $\mathrm{Cs}_{8}$ is not an exception and that the configuration of two layers starts at $n=19$. The geometries of homoatomic clusters (to be discussed in more detail below) are rather similar to those obtained by Manninen, ${ }^{18}$ minimizing the electrostatic interactions of point positive ions in a spherical and homogeneous negative background. This trend is, however, very different from that for $\mathrm{Na}_{n} \mathrm{Cs}_{n}$. In heteroclusters, the difference between the core radii of the $\mathrm{Na}$ and $\mathrm{Cs}$ atoms is responsible for (a) the formation of distinct homoatomic layers, which we interpret as a precursor of the order- 


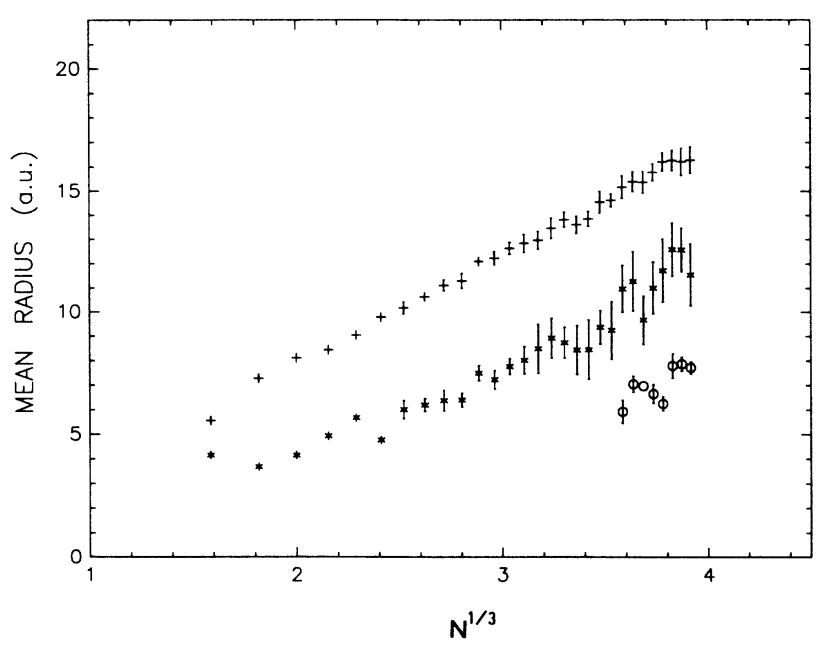

FIG. 2. Average radii of $\mathrm{Cs}(+$ and $O)$ and $\mathrm{Na}(*)$ shells and their widths (measured by the standard deviation) in $\mathrm{Na}_{n} \mathrm{Cs}_{n}$ vs $N^{1 / 3}$. $N=2 n$ is the number of atoms in the cluster.

ing effects found in the bulk solid alloy, and (b) the strong segregation of Cs atoms to the surface. Segregation is evident since the outer layer is always formed by Cs atoms. Segregation of Cs to the surface of very large $\mathrm{Na} / \mathrm{Cs}$ clusters was also obtained in the Monte Carlo simulations of Rice, ${ }^{8}$ as well as layer stratification near the surface.

Anagnostatos ${ }^{19}$ has proposed a way of interpreting the abundance spectrum and magic numbers of alkali-metal clusters based on geometrical considerations. Compared with our results, there is, however, an essential difference in the pattern of cluster growth, both for homoatomic and heteroatomic clusters. In the model proposed by Anagnostatos, the clusters grow outwards; that is, the external layers form over the previously formed inner layers. In contrast, our results indicate a strong reconstruction of the whole cluster as it grows. In the size range covered by our calculations, additional inner shells grow

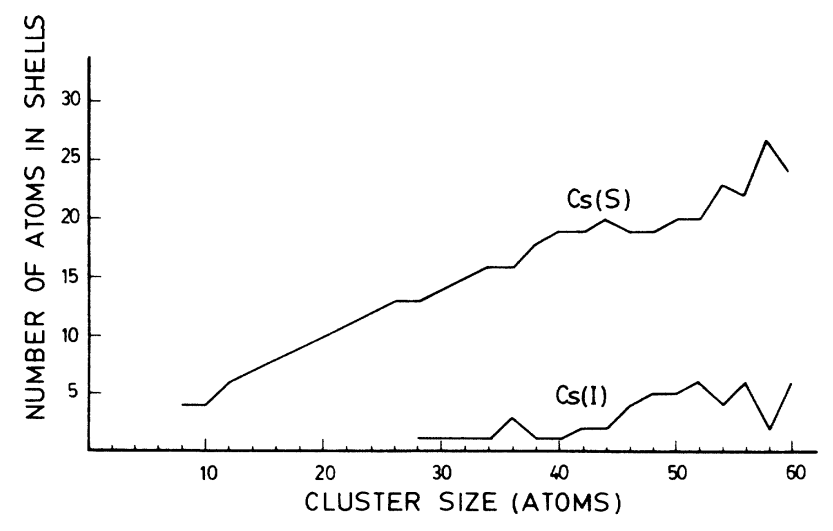

FIG. 3. Number of atoms in the inner $(I)$ and surface $(S)$ Cs shells of $\mathrm{Na}_{n} \mathrm{Cs}_{n}$ as a function of size. Note that these clusters have an even number of atoms. when the external shell is sufficiently filled. We may interpret this effect as a tendency to minimize the surface energy of the cluster. A particularly evident manifestation of this effect can be seen in Fig. 3, where, in the range $N=42-52$, the population of the surface shell remains nearly constant, while the additional Cs atoms give rise to an internal Cs layer.

Summarizing the features exposed so far, we identify three main conclusions: (a) First of all, in the small size regime considered in this paper, the geometries bear no relation to those of bulk crystals, since the surface effect is very strong and dominates the cluster reconstruction as $N$ grows. (b) Cs atoms strongly prefer the outer part of the cluster. This is again a surface-controlled effect, since Cs segregation lowers the surface energy of the cluster. (c) However, when the cluster is large enough, Cs atoms begin to form a new shell in the inner part of the cluster, and for $\mathrm{Na}_{60} \mathrm{Cs}_{30}$ we have found a sequence of layers $\mathrm{Na}$ $\mathrm{Cs}-\mathrm{Na}$-Cs. We interpret this as a precursor of the tendency to superlattice formation in the bulk solid alloy, due mostly to atomic size difference.

At this point it is fair to point out that in the process of calculating the equilibrium geometry of $\mathrm{Na}$-Cs clusters (also of pure $\mathrm{Na}_{n}$ or $\mathrm{Cs}_{n}$ ), we find a large number of relative minima with energies close to that of the ground state. This is a manifestation of the "soft" nature of clusters formed by alkali metals. This softness accounts for the strong reconstruction with increasing size, and it also suggests that very large cluster sizes are needed to exhibit the crystallographic structure of the bulk solid. This contrasts with the situation for clusters with ionic bonding. In a study of $\mathrm{Cs}_{n} \mathrm{O}$, we have found that the coordination around the oxygen impurity is rather stable as a function of cluster size, and furthermore, this coordination is similar to that in the bulk oxide. ${ }^{16}$

\section{B. Stability and magic numbers}

Sharp variations in the relative stability of the clusters as a function of size give rise to the so-called magic numbers, which appear as prominent features (maxima or pronounced steps) in the abundance spectrum.

Figure 4 gives the total energy per atom, $E(N) / N$, of

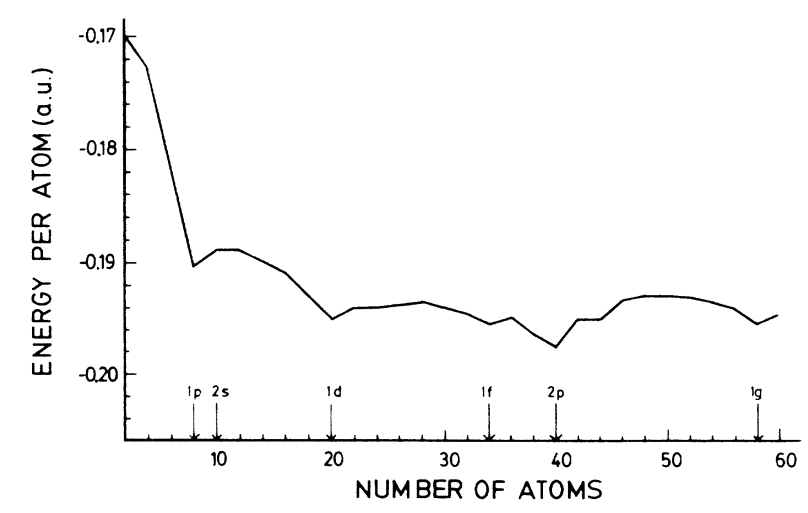

FIG. 4. Energy per atom, $E(N) / N$, in $\mathrm{Na}_{n} \mathrm{Cs}_{n}$ as a function of cluster size. Note that $N$ has only even values. Filling of electronic shells is indicated. 
$\mathrm{Na}_{n} \mathrm{Cs}_{n}$ as a function of the number of atoms in the cluster. $E(N) / N$ shows pronounced minima for $N=8,20$, 34,40 , and 58. This is a consequence of electronic shellclosing effects. Clusters with $8,20,34,40$, and 58 valence electrons have the closed-shell electronic configurations $1 s^{2} 1 p^{6}, \quad 1 s^{2} 1 p^{6} 2 s^{2} 1 d^{10}, \quad 1 s^{2} 1 p^{6} 2 s^{2} 1 d^{10} 1 f^{14}$, $1 s^{2} 1 p^{6} 2 s^{2} 1 d^{10} 1 f^{14} 2 p^{6}$, and $1 s^{2} 1 p^{6} 2 s^{2} 1 d^{10} 1 f^{14} 2 p^{6} 1 g^{18}$, respectively. We then predict that these clusters should be prominent in the abundance spectra. The general trend in the energy curve is similar to that obtained for homoatomic alkali-metal clusters both in the jellium and SAPS models, and the above numbers, $N=8,20,34,40$, and 58, are, in fact, experimental magic numbers of pure alkali-metal clusters ${ }^{1,2}$ and mixed alkali-metal cluster. ${ }^{20,21}$ We then conclude that the magic numbers of pure and mixed alkali-metal clusters depend only on the number of electrons in the cluster. However, the geometry and atomic distribution are sensitive to the nature of the atoms in the cluster and to their relative proportion.

The convergence of the energy of the cluster to the bulk limit can be studied by calculating the cohesive energy per atom. For the clusters with composition $\mathrm{Na}_{m} \mathrm{Cs}_{n}$, the cohesive energy per atom can be written

$$
\begin{aligned}
E_{\mathrm{coh}}\left(\mathrm{Na}_{m} \mathrm{Cs}_{n}\right)=\frac{1}{m+n}[ & m E(\mathrm{Na})+n E(\mathrm{Cs}) \\
& \left.-E\left(\mathrm{Na}_{m} \mathrm{Cs}_{n}\right)\right],
\end{aligned}
$$

where $E(\mathrm{Na})$ and $E(\mathrm{Cs})$ are free-atom energies calculated by the same model. The cohesive energies are plotted in Fig. 5 for $m=n$ and $2 n$. As mentioned above, the only bulk solid alloy is the ordered compound $\mathrm{Na}_{2} \mathrm{Cs}$. The

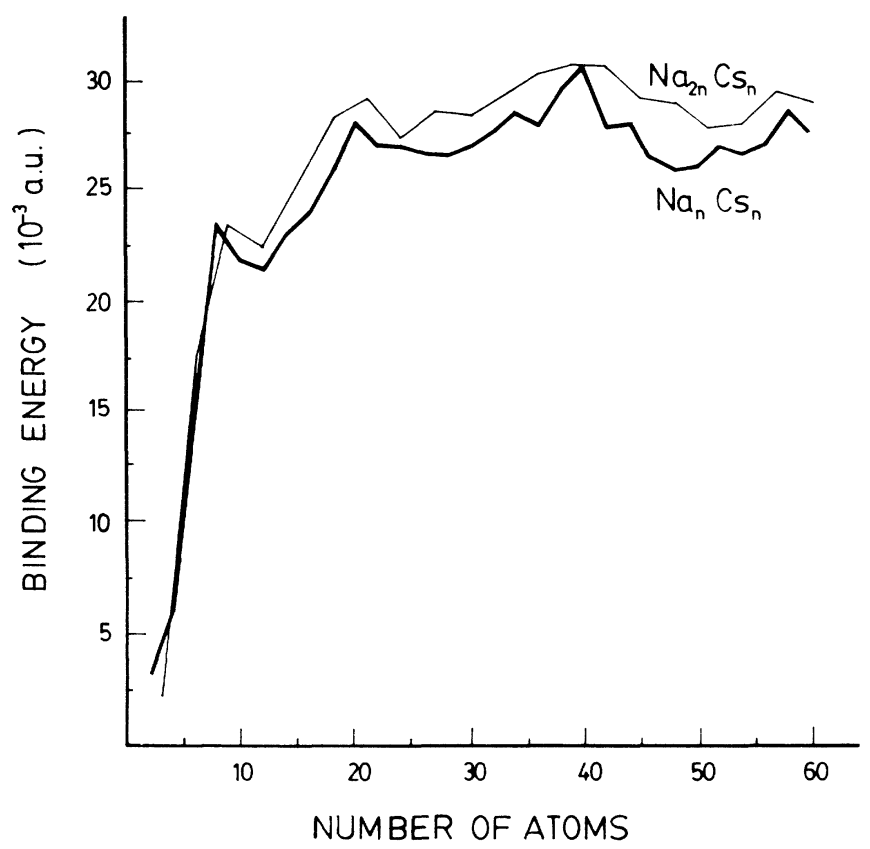

FIG. 5. Cohesive energy (per atom) of $\mathrm{Na}_{n} \mathrm{Cs}_{n}$ and $\mathrm{Na}_{2 n} \mathrm{Cs}_{n}$ vs cluster size $N(N=2 n$ or $3 n$, respectively). Note that only even values of $N$ occur in $\mathrm{Na}_{n} \mathrm{Cs}_{n}$ and only multiples of 3 in $\mathrm{Na}_{2 n} \mathrm{Cs}_{n}$. cohesive energy (per atom) of this compound is given by

$E_{\text {coh }}\left(\mathrm{Na}_{2}\right.$ Cs alloy $)=\frac{1}{3}\left[2 E_{\mathrm{coh}}(\mathrm{Na})+E_{\mathrm{coh}}(\mathrm{Cs})\right]+\Delta H$,

where $E_{\text {coh }}(\mathrm{Na})$ and $E_{\text {coh }}(\mathrm{Cs})$ are the cohesive energies of the pure metals, and $\Delta H$ is the heat of formation of the alloy with respect to the pure metals. $\Delta H$ is negative, since the compound exists, but its precise value has not yet been determined. The only theoretical calculation ${ }^{22}$ for this compound gives the wrong sign for $\Delta H$, but at least suggests that the absolute value of $\Delta H$ is small compared to the cohesive energies of the pure metals $\mathrm{Na}$ and Cs), which is the usual situation for metallic alloys. Additional evidence for a small $|\Delta H|$ comes from the experimental fact that the number of intermetallic compounds in this binary system is only one $\left(\mathrm{Na}_{2} \mathrm{Cs}\right)$. Consequently, we can approximate

$$
E_{\text {coh }}\left(\mathrm{Na}_{2} \mathrm{Cs} \text { alloy }\right) \approx \frac{1}{3}\left[2 E_{\mathrm{coh}}(\mathrm{Na})+E_{\mathrm{coh}}(\mathrm{Cs})\right] .
$$

Using experimental values for the cohesive energies of the pure metals, Eq. (4) gives $E_{\text {coh }}\left(\mathrm{Na}_{2} \mathrm{Cs}\right.$ alloy $)=0.037$ a.u. On the other hand, the cohesive energy of the cluster $\mathrm{Na}_{26} \mathrm{Cs}_{13}$ is already equal to 0.031 a.u./atom. From the evolution of $E_{\text {coh }}$ with cluster size in Fig. 5, we can conclude that the bulk limit of $E_{\text {coh }}$ is approached rather rapidly.

One could argue that a better idea of the convergence to the bulk limit can be obtained by using in Eq. (4) theoretical predictions for $E_{\text {coh }}(\mathrm{Na})$ and $E_{\text {coh }}(\mathrm{Cs})$ (obtained under the same assumptions used in our cluster calculations) instead of the experimental values. Nevertheless, it is well known that density-functional theory and the pseudopotential approximation work extremely well for the simple alkali metals and the theoretical predictions for the cohesive energy are in very good agreement with experiment. For instance, an all-electron density functional calculation by Moruzzi et al. ${ }^{23}$ gives $E_{\text {coh }}(\mathrm{Na})=1.116 \mathrm{eV} /$ atom, in nearly perfect agreement with the experimental value ${ }^{24}$ of $1.113 \mathrm{eV} /$ atom. Calculations using the empty-core pseudopotential ${ }^{25,26}$ also give very good results for the binding energies of $\mathrm{Na}$ and Cs. Actually, we estimate that the error from using experimental pure-metal cohesive energies instead of calculated ones is not larger than the error from neglecting $\Delta H$ in Eq. (3).

In Fig. 6 we give the energy balance of the reaction

$$
\mathrm{Na}_{2 n}+\mathrm{Cs}_{n} \rightarrow \mathrm{Na}_{2 n} \mathrm{Cs}_{n} .
$$

This reaction is exothermic; that is, the mixed cluster is stable with respect to the pure fragments, but the magnitude of the heat of the reaction, if measured per atom, decreases rapidly with increasing size. In the case $n \rightarrow \infty$ this energy balance becomes the energy of formation $\Delta H$ of the bulk $\mathrm{Na}_{2} \mathrm{Cs}$ compound starting from the pure metals, but at small $n$ the formation of the $\mathrm{Na}_{2 n} \mathrm{Cs}_{n}$ clusters does not proceed, evidently, through reaction (5). The heat of the reaction $\mathrm{Na}_{n}+\mathrm{Cs}_{n} \rightarrow \mathrm{Na}_{n} \mathrm{Cs}_{n}$ is also included in Fig. 6.

The evolution of the Kohn-Sham one-electron eigenval- 


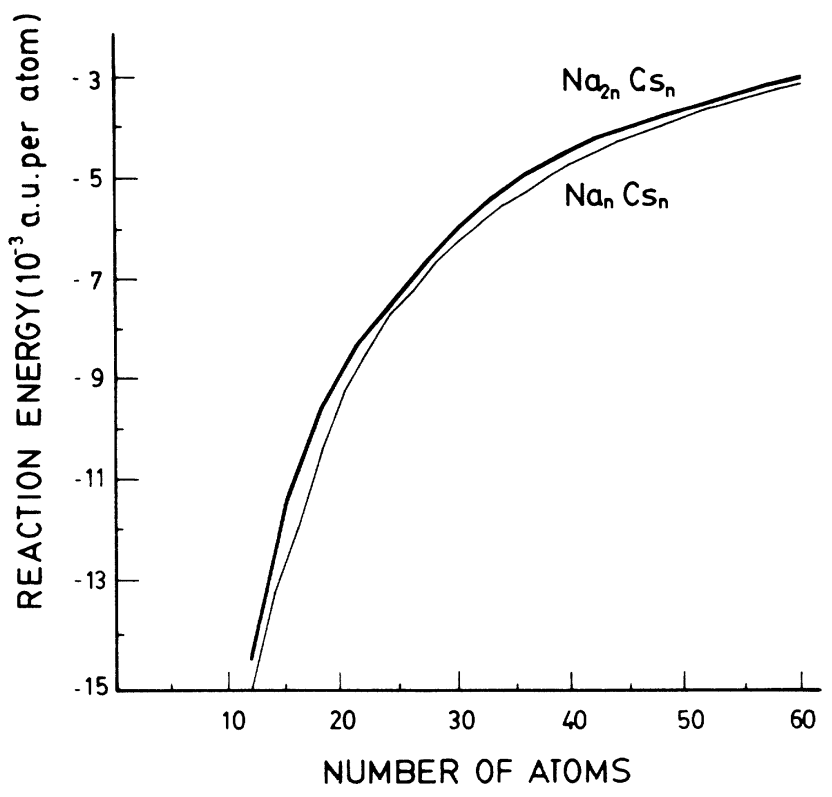

FIG. 6. Energy balance for the reactions $\mathrm{Na}_{2 n}+\mathrm{Cs}_{n}$ $\rightarrow \mathrm{Na}_{2 n} \mathrm{Cs}_{n}$ and $\mathrm{Na}_{n}+\mathrm{Cs}_{n} \rightarrow \mathrm{Na}_{n} \mathrm{Cs}_{n}$ vs $N(N=3 n$ or $2 n$, respectively).

ues ${ }^{12}$ for the ground state of $\mathrm{Na}_{n} \mathrm{Cs}_{n}$ is given in Fig. 7. One of the noticeable features of this figure is the behavior of the $1 d$ and $2 s$ levels. In the size range $N \leq 26$ these two levels are close in energy, although the $2 s$ is below the $1 d$ state. Then the levels cross and the $1 d$ state becomes lower in energy for $N \geq 28$. For comparison we notice that the $1 d$ state is always below the $2 s$ in the jellium model of pure alkaline-metal clusters. ${ }^{27}$ The peculiar behavior observed here for the mixed clusters is correlated with the nature of the atom that in most cases exists at the cluster center. This central atom is $\mathrm{Na}$ for $N \leq 26$, Cs between $N=28$ and 46, and $\mathrm{Na}$ again for $N>46$. A Na atom provides a more attractive potential than a Cs atom, giving rise to an enhanced probability for $s$-type electrons in the central region. For small clusters $(N \leq 26)$ this effect is strong enough to place the $2 s$ level below the $1 d$ level. Inversion between the $1 d$ and $2 s$ levels has also been found in the case of divalent impurities in alkali-metal clusters. ${ }^{28,29}$ This inversion is responsible for the appearance of a new magic number in those clusters, corresponding to ten electrons.

Also, the $2 p$ and $1 g$ states are closer here than in the jellium model for pure clusters. ${ }^{27}$ This effect is also present in recent jellium-on-jellium calculations for $\mathrm{Na}$ clusters coated by Cs. ${ }^{30}$ Finally, the oscillations of the $2 s$ eigenvalue in the region $N=50-58$ are correlated with the number of Cs atoms in the inner shell of the cluster.

\section{C. $\mathrm{Na}_{n}$ geometries}

It is the purpose of the next section (Sec. III D) to discuss the geometries of small $\mathrm{Na}_{m} \mathrm{Cs}_{n}$ clusters formed by an inner $\mathrm{Na}$ shell and and outer $\mathrm{Cs}$ shell. In particular, we wish to show the way the external Cs layer modifies the geometrical arrangement of the internal $\mathrm{Na}$ content with respect to the geometry of the pure $\mathrm{Na}_{n}$ counterpart.

As a prior step, we first discuss the geometries of the pure $\mathrm{Na}_{n}$ clusters, comparing these with the results of other calculations which avoid the spherical approximation for the total ionic potential. Because of this approximation, our resulting geometries for very small clusters are more spherical than those obtained in more accurate calculations, which are restricted, however, to date, to very small clusters. $A b$ initio configuration-interaction (CI), ${ }^{31}$ extended-Hückel, ${ }^{32}$ and density-functional pseudopotential $^{33-36}$ results are available for the equilibrium geometries of $\mathrm{Na}_{n}$ up to $n=9$. These are, as ours, static calculations. Our stable geometries for some clusters are given in the left-hand part of Fig. $8 . \mathrm{Na}_{5}$ is a trigonal bipyramid. This is a metastable geometry in Ref. 31 , lying

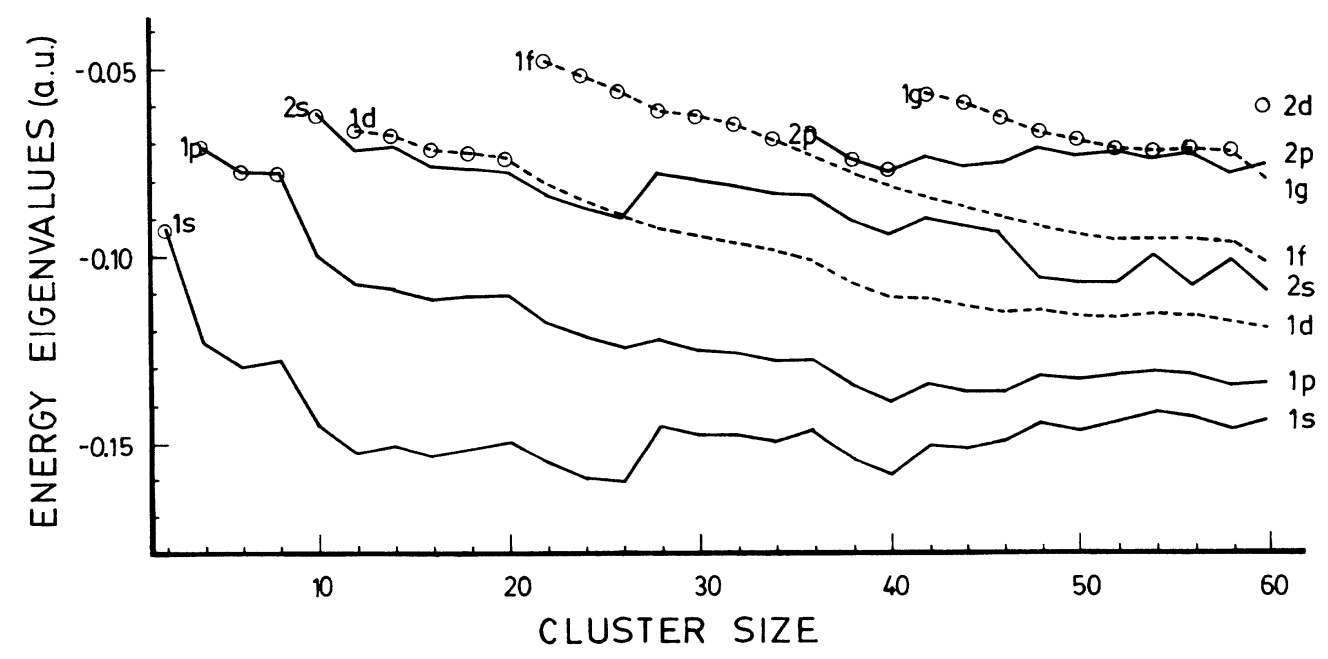

FIG. 7. One-electron Kohn-Sham eigenvalues as a function of cluster size for $\mathrm{Na}_{n} \mathrm{Cs}_{n}$. The outermost occupied level is indicated by a circle. 
$0.04 \mathrm{eV} /$ atom above the stable planar form. The geometry for both $\mathrm{Na}_{6}$ and $\mathrm{Na}_{7}$ is the regular octahedron, but with an atom in its center in the case of $\mathrm{Na}_{7} . \mathrm{Na}_{6}$ is a pentagonal pyramid and $\mathrm{Na}_{7}$ a pentagonal bipyramid in
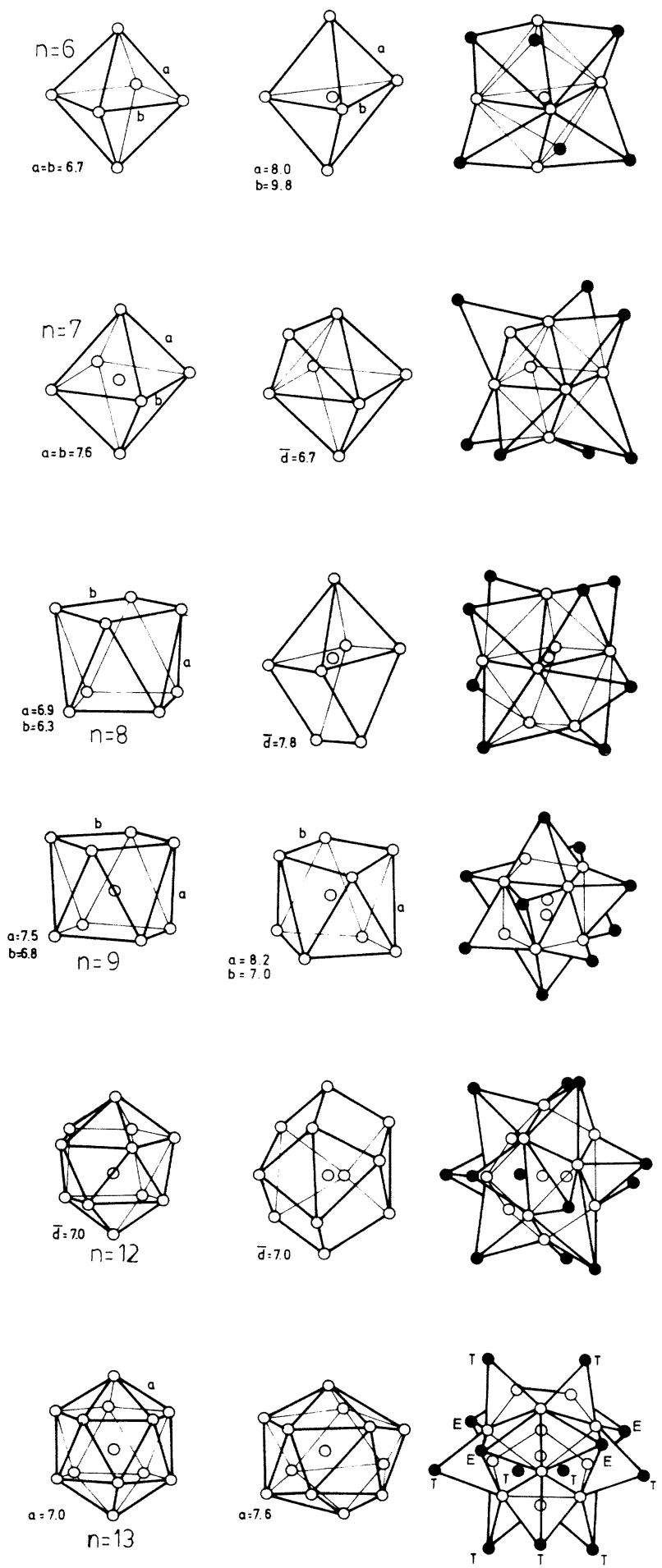

FIG. 8. Geometries of pure $\mathrm{Na}_{n}$ (left-hand column), $\mathrm{Na}_{n}$ part in $\mathrm{Na}_{n} \mathrm{Cs}_{n}$ (central column), and whole $\mathrm{Na}_{n} \mathrm{Cs}_{n}$ (right-hand column), for $n=6,7,8,9,12$, and 13. Open and solid circles represent $\mathrm{Na}$ and $\mathrm{Cs}$ atoms, respectively. Distances are all in a.u. more accurate calculations. ${ }^{31-36}$ We have found that the pentagonal bypyramid is the first excited local minimum of $\mathrm{Na}_{7}$ in the SAPS model, being almost degenerate with the centered octahedron (the difference in energy is only $0.004 \mathrm{eV} /$ atom, whereas the cohesive energy per atom is $0.65 \mathrm{eV}$ ). Our geometry for $\mathrm{Na}_{8}$ is a square antiprism, in agreement with the density functional results of Manninen and co-workers ${ }^{34,36}$ and Arvati et al. ${ }^{35}$ This occurs because in the later calculations the electronic configuration is close to $1 s^{2} 1 p^{6}$, which gives a spherically symmetric electron density to which the ionic lattice responds, adopting also a very spherical geometry. $\mathrm{Na}_{9}$ is an atom-centered square antiprism. The more stable geometry obtained by Wang et al. ${ }^{32}$ and Cogordan et al. is, in our calculations, a local minimum, $0.03 \mathrm{eV} /$ atom above the absolute minimum. The equilibrium geometries of $\mathrm{Na}_{10}$ and $\mathrm{Na}_{11}$ are very regular. $\mathrm{Na}_{10}$ is a centered tricapped trigonal prism $\left(D_{3 h}\right)$, and $\mathrm{Na}_{11}$ is a centered square antiprism bicapped at the square faces $\left(C_{44^{\prime}}\right) . \mathrm{Na}_{12}$ is a distorted centered icosahedron with a vertex lost and $\mathrm{Na}_{13}$ a perfect centered icosahedron. A configuration-interaction calculation by Pacchioni and Koutecky ${ }^{37}$ suggested that the equilibrium geometry of $\mathrm{Li}_{13}$ is a centered icosahedron. A more complete dynamical study has been performed by Ballone et al. ${ }^{10}$ for $\mathrm{Na}_{20}$ at $T=0$ and $200 \mathrm{~K}$. The equilibrium geometry at $T=200$ $\mathrm{K}$ (in an average sense) resembles our static geometry (see Fig. 9) in that there are two atoms inside a more external arrangement of the remaining 18 atoms.

In summary, we have found that the equilibrium geometries predicted by the SAPS model for small $\mathrm{Na}$ clusters are often identical to those of more accurate calculations and, in other cases, coincide with the geometries of low-lying isomers. Since, first of all, the SAPS model is expected to become better for larger clusters (which are more spherical than the smaller ones) and, on the other hand, the energy differences between the ground-state equilibrium geometry and those of low-lying isomers are rather small, we can have some confidence in our predictions for "large" mixed clusters.

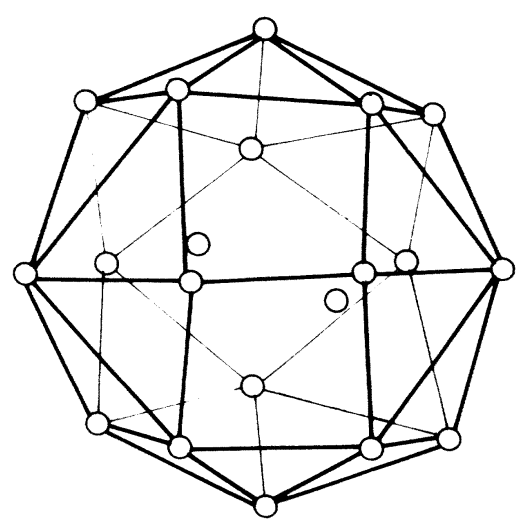

$\mathrm{Na}_{20}$

FIG. 9. Equilibrium geometry of pure $\mathrm{Na}_{20}$. 


\section{Geometries of $\mathrm{Na}_{n} \mathrm{Cs}_{n}$}

A few representative clusters (corresponding to $n=6$, $7,8,9,12$, and 13) have been chosen as illustrative examples of the small size range. Figure 8 shows, for each $n$, the geometry of pure $\mathrm{Na}_{n}$ (left-hand part), that of the $\mathrm{Na}$ core (denoted $\mathrm{Na}_{n}^{*}$ ) in the $\mathrm{Na}_{n} \mathrm{Cs}_{n}$ heterocluster (middle column), and the whole $\mathrm{Na}_{n} \mathrm{Cs}_{n}$ geometry (right-hand column). The pure $\mathrm{Na}_{n}$ geometries have already been discussed in Sec. III C. $\mathrm{Na}_{6}^{*}$ is a nonregular centered trigonal bipyramid $\left(D_{3 h}\right)$. This is a polyhedron with six faces, to which the six outer Cs atoms of $\mathrm{Na}_{6} \mathrm{Cs}_{6}$ can be easily coordinated. Each Cs atom is on top of one of the faces, and the six Cs atoms then form a trigonal prism surrounding the $\mathrm{Na}_{6}^{*}$ core. $\mathrm{Na}_{7}^{*}$ brings out the central atom of $\mathrm{Na}_{7}$ on top of a face of the octahedron. The seven Cs atoms are then on top of the remaining seven faces of the octahedron. The square antiprism $\mathrm{Na}_{8}$ reconstructs in such a way that $\mathrm{Na}_{8}^{*}$ is a centered octahedron with one vertex split into two symmetrical ones and $C_{2 v}$ symmetry, as the way to provide the eight faces needed to host the eight $\mathrm{Cs}$ atoms. The $\mathrm{Na}_{9}$ structure (centered square antiprism) remains the same in forming the heterocluster, and the nine Cs atoms become coordinated to nine of the ten faces, leaving one triangular face empty. Note, however, that the interatomic distances in the $\mathrm{Na}_{9}^{*}$ core have increased with respect to those in the free $\mathrm{Na}_{9}$ cluster. The structures of the $\mathrm{Na}_{10}^{*}$ and $\mathrm{Na}_{11}^{*}$ cores are different from those of the pure $\mathrm{Na}$ clusters, the trend governing the changes being the tendency to form a polyhedron with a number of faces closer to the number of Cs atoms. The $\mathrm{Na}_{12}^{*}$ core in $\mathrm{Na}_{12} \mathrm{Cs}_{12}$ has $D_{3 h}$ symmetry; it is formed by one $\mathrm{Na}$ atom at the center of a polyhedron with 12 faces. Each of the 12 Cs atoms is coordinated to one of the 12 faces of the $\mathrm{Na}$ polyhedron, preserving the $D_{3 h}$ symmetry. Finally, $\mathrm{Na}_{13}^{*}$ is a little dis- torted with respect to $\mathrm{Na}_{13}$, but it remains as an icosaedral arrangement, covered by $13 \mathrm{Cs}$ atoms in the following way: Four Cs atoms (atoms $E$ ) are on top of edges of the $\mathrm{Na}_{13}^{*}\left(I_{h}\right)$ core, and each one is coordinated to four $\mathrm{Na}$ atoms. The remaining nine $\mathrm{Cs}$ atoms are on top of nine triangular faces (atoms $T$ ), whereas three faces rest empty.

Interatomic distances are also given for $\mathrm{Na}_{n}$ and $\mathrm{Na}_{n}^{*}$ in Fig. 8. When only one distance is reported, this means that the polyhedron is regular or that an average $(\bar{d})$ of the polyhedron edges is made. Averaged values for the edges of the external polyhedra joining $\mathrm{Cs}$ and $\mathrm{Na}$ atoms are between 8.1 and 8.3 a.u. for the clusters given in Fig. 8.

From the analysis of the cluster topologies, we conclude that the geometry of the $\mathrm{Na}_{n}^{*}$ core in $\mathrm{Na}_{n} \mathrm{Cs}_{n}$ is different from that of free $\mathrm{Na}_{n}$, and that the mechanism of reconstruction is the formation of a polyhedron with a number of faces consistent with the number of external Cs atoms. Then these Cs atoms can sit on top of those faces. This result indeed suggests that specially stable clusters will be obtained by starting with the equilibrium polyhedron for $\mathrm{Na}_{n}$ and capping each face with a Cs atom. Note, however, that the geometrical stabilization effects are expected to be very small in pure and mixed alkali-metal clusters and will be unobservable in the usual mass spectra. By looking again at Fig. 4, we note that the stability peaks of $\mathrm{Na}_{n} \mathrm{Cs}_{n}$ clusters $(N=8,20,34,40$, and 58) correspond to electronic shell-closing effects.

\section{ACKNOWLEDGMENTS}

This work has been supported by Dirección General de Investigación Científica y Tecnica (Grant No. PB 860654-C02), Junta de Castilla y León, and Universidad de Valladolid (Consejo Social).
${ }^{1}$ W. A. de Heer, W. D. Knight, M. Y. Chou, and M. L. Cohen, in Electronic Shell Structure and Metal Clusters, Vol. 40 of Solid State Physics, edited by H. Ehrenreich, F. Seitz, and D. Turnbull (Academic, New York, 1987), p. 93.

${ }^{2}$ M. Y. Chou, A. Cleland, and M. L. Cohen, Solid State Commun. 52, 645 (1984).

${ }^{3}$ K. Clemenger, Phys. Rev. B 32, 1359 (1985).

${ }^{4}$ W. Ekardt and S. Penzar, Phys. Rev. B 38, 4273 (1988).

${ }^{5}$ J. Gryko and S. A. Rice, J. Phys. F 12, L245 (1982).

${ }^{6}$ M. P. D'Evelyn and S. A. Rice, J. Chem. Phys. 78, 5225 (1983).

${ }^{7}$ J. H. Harris, J. Gryko, and S. A. Rice, J. Chem. Phys. 87, 3069 (1987).

${ }^{8}$ S. A. Rice, Z. Phys. Chem. [Neue Folge] 156, S53 (1988).

${ }^{9}$ M. J. López, A. Mañanes, J. A. Alonso, and M. P. Iñiguez, Z. Phys. D 12, 237 (1989)

${ }^{10} \mathrm{P}$. Ballone, W. Andreoni, R. Car, and M. Parrinello, Europhys. Lett. 8, 73 (1989).

${ }^{11}$ M. Hansen and K. Anderko, Constitution of Binary Alloys (McGraw-Hill, New York, 1958).

${ }^{12}$ W. Kohn and L. J. Sham, Phys. Rev. 140, A1133 (1965).

${ }^{13}$ Theory of the Inhomogeneous Electron Gas, edited by $\mathbf{N} . \mathbf{H}$. March and S. Lundqvist (Plenum, New York, 1983).
${ }^{14}$ N. W. Ashcroft, Phys. Lett. 23, 48 (1966).

${ }^{15}$ M. P. Iñiguez, M. J. López, J. A. Alonso, and J. M. Soler, Z. Phys. D 11, 163 (1989).

${ }^{16}$ U. Lammers, A. Mañanes, G. Borstel, and J. A. Alonso, Solid State Commun. 71, 591 (1989).

${ }^{17} \mathrm{Na}_{7}$ was reported in Ref. 15 as an "empty" cluster. A more extensive search has shown that it contains a central atom.

${ }^{18}$ M. Manninen, Solid State Commun. 59, 281 (1986).

${ }^{19}$ G. S. Anagnostatos, Phys. Lett. A 128, 266 (1988).

${ }^{20}$ M. M. Kappes, M. Schär, and E. J. Schumacher, J. Phys. Chem. 91, 658 (1987).

${ }^{21}$ C. Brechignac and P. H. Cahuzac, Z. Phys. D 3, 121 (1987).

22J. Hafner, Phys. Rev. B 15, 617 (1977).

${ }^{23}$ V. L. Moruzzi, J. F. Janak, and A. R. Williams, Calculated Electronic Properties of Metals (Pergamon, New York, 1978).

${ }^{24}$ C. Kittel, Introduction to Solid State Physics, 5th ed. (Wiley, New York, 1976).

${ }^{25}$ N. W. Ashcroft and D. C. Langreth, Phys. Rev. 155, 682 (1967).

${ }^{26}$ J. Häfner, in From Hamiltonians to Phase Diagrams, Vol. 70 of Springer Series in Solid State Sciences, edited by Peter Fulde (Springer-Verlag, Berlin, 1987). 
${ }^{27}$ W. Ekardt, Phys. Rev. B 29, 1558 (1984).

${ }^{28}$ C. Baladron and J. A. Alonso, Physica B 154, 73 (1988).

${ }^{29}$ W. Pewestorf, V. Bonacić-Koutecký, and J. Koutecký, J. Chem. Phys. 89, 5794 (1988).

${ }^{30}$ W. Ekardt, Phys. Rev. B 34, 526 (1986).

${ }^{31}$ V. Bonacic-Koutecký, P. Fantucci, and J. Koutecký, Phys. Rev. B 37, 4369 (1988).

${ }^{32}$ Y. Wang, T. F. George, D. M. Lindsay, and A. C. Beri, J. Chem. Phys. 86, 3493 (1987).

${ }^{33}$ J. L. Martins, J. Buttet, and R. Car, Phys. Rev. B 21, 1804
(1985).

${ }^{34}$ M. Manninen, in Condensed Matter Theories, Vol. 3, edited by J. S. Arponen, R. F. Bishop, and M. Manninen (Plenum, New York, 1988), p. 221.

${ }^{35}$ S. Arvati, L. F. Dona dalle Rose, P. L. Silvestrelli, and F. Toigo, Nuovo Cimento D 11, 1063 (1989).

36J. A. Cogordan, R. Virkkunen, and M. Manninen, Phys. Scr. 38, 758 (1988).

${ }^{37}$ G. Pacchioni and J. Koutecký, J. Chem. Phys. 73, 4511 (1980). 